\title{
Downlink LTE System Performance Improvement by Using BCH Codes over LTE-MIMO Channel
}

\author{
Ghasan A. Hussain*, Lukman Audah*
}

\author{
*Wireless and Radio Science Centre (WARAS), Faculty of Electrical and Electronic Engineering, \\ Universiti Tun Hussein Onn Malaysia, Parit Raja, 86400 Batu Pahat, Johor, MALAYSIA
}

Received 20 May 2018; accepted 24 June 2018, available online 6 August 2018

\begin{abstract}
Long-term Evolution (LTE) has potential feasibility to implement high-speed data transmission. This technology is essential for accommodating the rising utilization of data and voice service. Nonetheless, the main problem is the deteriorated performance of wireless communication systems following the elevation in bit error rate (BER) owing to multipath fading environments. Therefore, using channel coding is essential to reduce BER, which leads to enhancement of system performance of digital communication systems. Previous studies have reported utilization of channel codes including turbo and convolutional codes in LTE systems for attaining error control system. As such, the key challenge of selecting category of coding method is the trade-off between elevating decoding complexity and enhancing system performance. Hence, the study herein proposed Bose and Chaudhuri $(\mathrm{BCH})$ codes as channel coding for downlink LTE system over MIMO channel. This paper is contributing to attain an adaptive error control in Downlink LTE system over LTE-MIMO channel by using BCH codes that have low decoding complexity compared to turbo and convolutional codes that have been suggested in previous studies, as well as the purpose of using MIMO channel is to increase the system capacity and thus improve the LTE system performance. The findings indicated that BCH codes had reduced BER better than both of turbo and convolutional codes in both quadrature phase-shift keying (QPSK) modulation schemes and binary phase-shift keying (BPSK) with regard to the downlink LTE system performance. Where, the suggested method had approximately $4 \mathrm{~dB}$ and $6 \mathrm{~dB}$ coding gain at $1 \times 10^{-1}$ against turbo and convolutional codes respectively, in BPSK. While, for QPSK, the proposed method had approximately $4 \mathrm{~dB}$ compared to both of turbo and convolutional codes. Nonetheless, enhanced performance of the suggested system was observed through reducing BER of the received data by adding the number of antennas in MIMO channel.
\end{abstract}

\section{Introduction}

The main goal of fourth generation (4G) mobile communication is to deliver high-speed data transmission. This demands higher charge to improve the technology standards. The additional cost may lower the consumer's attraction. The standard of $4 \mathrm{G}$ mobile communication is known as Universal Mobile Telecommunications System (UMTS) Terrestrial Radio Access Network (E-UTRAN), which was initiated by Third Generation Partnership Project (3GPP). Unlike third-generation $(3 \mathrm{G})$ standard that uses CDMA schemes, the LTE uplink transmission utilizes single carrierfrequency division multiple access (SC-FDMA) and orthogonal frequency division multiplexing (OFDM) intended for downlink [1].

Fig. 1 illustrates the network structural design of LTE that is known as System Architecture Evolution (SAE). The SAE contains two main components namely E-UTRAN and Evolved Packed Core (EPC), both of EPC and E-UTRAN together compose a system known as Evolved Packet System (EPS). From packet data network gateway (P-GW) to user equipment (UE), IP packets are routed by EPS with a recognized quality of service (QoS).
Meanwhile, E-UTRAN provide transmission data security through managing the radio resources [2].

The E-UTRAN merely includes base stations that are combined with UEs for achieving the functions of networks air interface. Moreover, the flat structural design of E-UTRAN. Hence, there is no centralized controlling in E-UTRAN [3]. Furthermore, the EPC network is linked to E-UTRAN over standard interfaces [4]. The EPC including internet coupled with UE allow exchanging of data packets and retaining QoS. The EPC contains policy control and charging rules function (PCRF), mobility management entity (MME), home subscriber service (HSS), P-GW and serving gateway (SGW) [1].

Initially, OFDM was proposed by Chang 1970 to mitigate the inter-symbol interference (ISI) and interchannel interference (ICI), where it was known as a digital modulation method [5]. The attribute of an OFDM and its utilization transform $3 \mathrm{G}$ to $4 \mathrm{G}$ in which application of multicarrier modulation (MCM) by means of $4 \mathrm{G}$ provides dual usages. Firstly, it reduces the noise or interference in the receiver of MCM system in comparison to single carrier modulation (SCM). Secondly, it mitigates fading channel and impulse noise in the long symbol period in MCM [5]. 


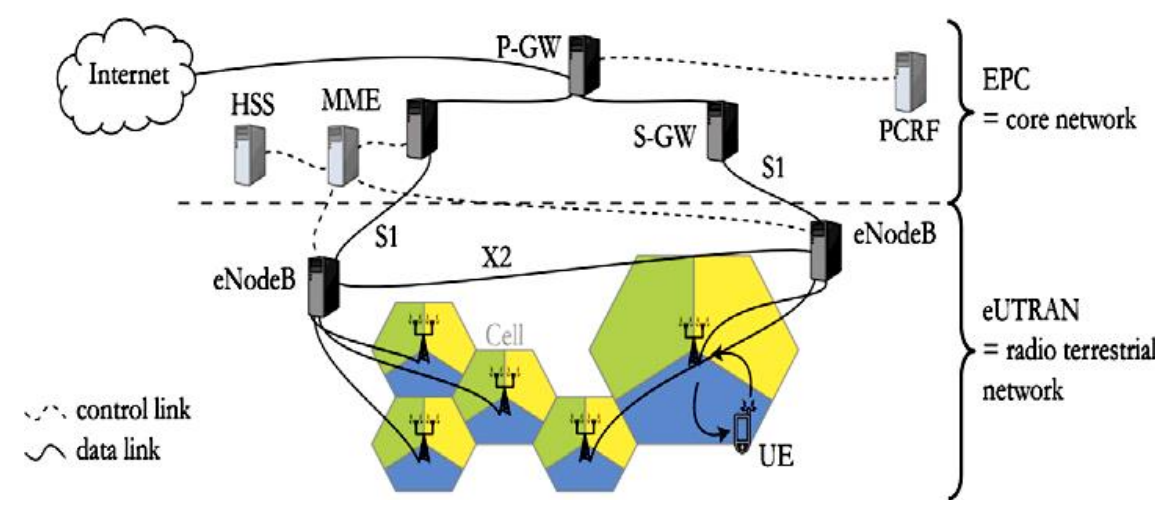

Fig. 1 LTE System Architecture Evolution (SAE).

The merging of (4X4) multi-input multi-output (MIMO) system and OFDM modulation was proposed to attain the specifications of 3GPP LTE and LTE-A. All of the power and latency limitations in addition to complexity and high performance that required to achieved was the challenging of research in mobile communication systems. They concluded there are a big flexibility of this system and achieving a good enhancement if exploit multi antenna techniques [6] Primarily, the merging of MIMO with OFDM systems have been applied widely in 4G wireless systems [7] Moreover, incorporation of MIMO-OFDM with LTE will elevates throughput by MIMO, whereas the frequency selective fading will be adapted by OFDM system to manifold flat fading sub-channels that can be equalized straightforwardly. $2 \times 2$ MIMO is proposed in this research to be used with LTE system as mandatorily support. The performance of two transmitter and receiver antennas has been presented over frequency selective fading environment. The results show that; the performance of proposed LTE system is better than of single antenna and give more throughput [7].

The channel coding is among the most important issues of digital communication systems. The automatic repeat request (ARQ) and forward error correction (FEC) are the two categories of error correction methods. In the case that errors identified in ARQ method, the mechanism of error detection is applied to enable data packets retransmission to the receiver (by requesting from the receiver). On the other hand, the redundancy bits will be supplemented to FEC method to the data bits that is performed through convolutional or block wise (known as block coding). The aforementioned coded bit relies on the two former bits and present data bit. The two methods of convolutional codes and block codes are applied in LTE [8]. The 1/3 code rate of convolutional code has been applied to construct a hardware structural design of LTE system based on 3GPP standard by applying MATLAB simulation model as described in a previous study [9]. The findings indicate that convolutional code resulted in the enhancement of their system performance in comparison with performance of the un-coded system. Nevertheless, the decoding procedure of convolutional codes is challenging, particularly higher complexity is observed when the data length is augmented [10]. On the other hand, a previous study has proposed turbo codes aimed at 3GPP LTE [11,12]. Due to the instability of decoding outcomes, it is crucial to attain the stability amongst the performance of decoding and the complexity of the implementation [11].

Hence, Bose and Chaudhuri (BCH) code has been proposed in the current study to be utilized in downlink LTE system across LTE-MIMO channel to attain further enhancement and lower complexity compared to other coding methods including convolutional and turbo codes $[13,14]$.

\section{Proposed System}

The suggested system is illustrated in Fig. 2. The parameters that have been applied in this study are based on the LTE parameters [15] as demonstrated in Table 1. The Downlink LTE system performance has been simulated using MATLAB software over multipath LTEMIMO channel and tested by using two modulation schemes BPSK and QPSK. The novelty in this paper by using $\mathrm{BCH}$ code in Downlink LTE system in channel coding to achieve the reliability with low decoding complexity.

Table 1: LTE System Parameters SIMULATION ENVIRONMENT OF LTE SYSTEM

\begin{tabular}{lc} 
OCCUPIED SUB-CARRIER & $20 \mathrm{MHz}$ \\
\hline CHANNEL & $\begin{array}{c}\text { LTE-MIMO Channe } \\
\text { Multipath Fading } \\
\text { Channel }\end{array}$ \\
NUMBER OF IFFT/FFT POINTS & 2048 \\
NO. OF OCCUPIED SUB-CARRIER & 1200 \\
CYCLIC PREFIX LENGTH & 144 \\
MODULATION & BPSK and QPSK \\
SAMPLING RATE & $30.72 \mathrm{MHz}$ \\
ERROR CORRECTION & BCH Codes \\
TECHNIQUES &
\end{tabular}
TECHNIQUES 


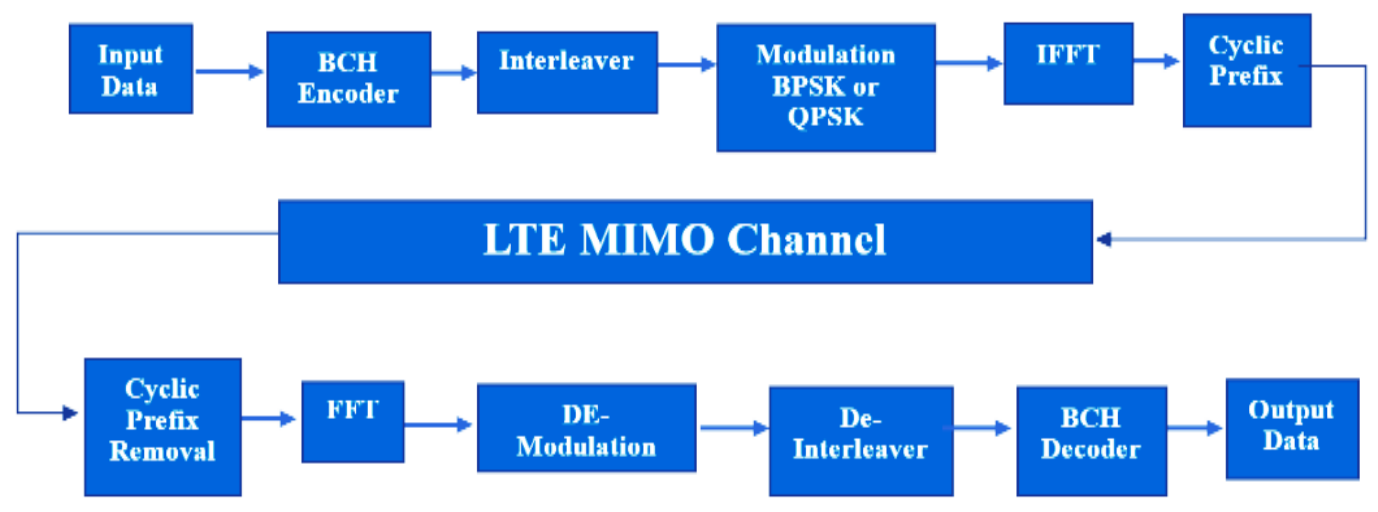

Fig. 2 Downlink LTE System over LTE-MIMO channel by using BCH code

The important parts of own proposed system have been explained in details as following:

\subsection{BCH Code}

The $\mathrm{BCH}$ codes was introduced by Bose, RayChoudhuri and Hocquenghem [16,17 and 18]. BCH codes are cyclic and linear codes, which are among the important error correcting codes [19]. The binary $\mathrm{BCH}$ codes have been demonstrated as a well-established technique for detecting errors compared to peripheral status, which contains infinity length (n) and minimum distance (d). When, in these circumstances, the long codes are unable to identify the errors in the transmission channel [20].

Assuming that $\mathrm{C}$ is a linear $\mathrm{BCH}$ block code, which is described by $\mathrm{n}$ that is specified by block length and $\mathrm{k}$ that is denoted by code dimension as (n, k) code. The code rate is denoted as $(\mathrm{r}=\mathrm{k} / \mathrm{n})$, owing to the channel coding, where the data rate is reduced by a factor of $r$. Any $\mathrm{BCH}$ code is characterized as described below [21].

Definition. $n=2^{m}-1, m \geq 3 . n-k \leq m t$ where; $m$ is any integer value and $t$ is error correcting capability, $t=\left[\left(d_{\min }-1\right) / 2\right]$ Since, $d_{\min }$ is the minimum hamming distance and it can correct t of errors per word [21]. The BCH of $(15,5)$ has been applied in this study.

In binary field GF (2), the Polynomials are defined and contains roots which belong to Galois field $\mathrm{GF}\left(2^{\mathrm{m}}\right)$. For instance, pi $(\mathrm{X})=1+\mathrm{X}^{3}+\mathrm{X}^{4}$ is an irreducible polynomial over GF (2) where there are no roots in this field. However, in the $\mathrm{GF}\left(2^{4}\right)$ has four roots. Table 2 is shown polynomials after replacing it's $X$ variables by the elements of the $\mathrm{GF}\left(2^{4}\right)$. It is proved that the polynomial has the roots $\alpha^{7}, \alpha^{11}, \alpha^{13}$ and $\alpha^{14}$. [22].
Table 2: GF $\left(2^{4}\right)$ that generated by $\mathrm{pi}(\mathrm{X})=1+\mathrm{X}+\mathrm{X}^{4}$

\begin{tabular}{|c|c|c|}
\hline $\begin{array}{c}\text { Exp. } \\
\text { Representation }\end{array}$ & $\begin{array}{c}\text { Polynomial } \\
\text { Representation }\end{array}$ & $\begin{array}{c}\text { Vector } \\
\text { Representation }\end{array}$ \\
\hline 0 & 0 & 0000 \\
\hline 1 & 1 & 1000 \\
\hline$\alpha$ & $\alpha$ & 0100 \\
\hline$\alpha^{2}$ & $\alpha^{2}$ & 0010 \\
\hline$\alpha^{3}$ & $\alpha^{3}$ & 0001 \\
\hline$\alpha^{4}$ & $1+\alpha$ & 1100 \\
\hline$\alpha^{5}$ & $\alpha+\alpha^{2}$ & 0110 \\
\hline$\alpha^{6}$ & $\alpha^{2}+\alpha^{3}$ & 0011 \\
\hline$\alpha^{7}$ & $1+\alpha+\alpha^{3}$ & 1101 \\
\hline$\alpha^{8}$ & $1+\alpha^{2}$ & 1010 \\
\hline$\alpha^{9}$ & $\alpha+\alpha^{3}$ & 0101 \\
\hline$\alpha^{10}$ & $1+\alpha+\alpha^{2}$ & 1110 \\
\hline$\alpha^{11}$ & $\alpha+\alpha^{2}+\alpha^{3}$ & 0111 \\
\hline$\alpha^{12}$ & $1+\alpha+\alpha^{2}+\alpha^{3}$ & 1111 \\
\hline$\alpha^{13}$ & $1+\alpha^{2}+\alpha^{3}$ & 1011 \\
\hline$\alpha^{14}$ & $1+\alpha^{3}$ & 1001 \\
\hline & & \\
\hline
\end{tabular}

Suppose $\varphi(X)$ is the minimal polynomial of Galois field $\mathrm{GF}\left(2^{\mathrm{m}}\right)$ in terms of elements $\beta$.

$$
\varphi(X)=\prod_{i=0}^{e-1}\left(x+\beta^{2}\right)
$$

where, $e$ is the smallest integer number when $\beta^{2 \mathrm{t}}=\beta$.

Table 3: All elements (Minimal polynomials) of the GF $\left(2^{4}\right)$ that generated by $\mathrm{pi}(\mathrm{X})=1+\mathrm{X}+\mathrm{X}^{4}$

\begin{tabular}{|c|c|}
\hline Conjugate Roots & Minimal Polynomials \\
\hline 0 & $\mathrm{X}$ \\
\hline 1 & $1+\mathrm{X}$ \\
\hline$\alpha, \alpha^{2}, \alpha^{4}, \alpha^{8}$ & $1+\mathrm{X}+\mathrm{X}^{4}$ \\
\hline$\alpha^{3}, \alpha^{6}, \alpha^{9}, \alpha^{12}$ & $1+\mathrm{X}+\mathrm{X}^{2}+\mathrm{X}^{3}+\mathrm{X}^{4}$ \\
\hline$\alpha^{5}, \alpha^{10}$ & $1+\mathrm{X}+\mathrm{X}^{2}$ \\
\hline$\alpha^{7}, \alpha^{11}, \alpha^{13}, \alpha^{14}$ & $1+\mathrm{X}^{3}+\mathrm{X}^{4}$ \\
\hline
\end{tabular}

Consider $\alpha$ is the primitive element of $\mathrm{GF}(24)$, from Table $3\left(1+\alpha+\alpha^{4}=0\right)$. The $\alpha, \alpha^{3}$ and $\alpha^{5}$ respectively are the minimal polynomials as shown in Table 3 [22].

$$
\begin{gathered}
\varphi_{1}(X)=1+X+X^{4} \\
\varphi_{3}(X)=1+X+X^{2}+X^{3}+X^{4} \\
\varphi_{5}(X)=1+X+X^{2}
\end{gathered}
$$


$\mathrm{BCH}(15,5)$ code has the capability of correcting $(\mathrm{t}=3)$ or less with $\mathrm{dmin} \geq 7$. The generator polynomial of this code (weight is 7) as following [22]:

$$
\begin{gathered}
\quad \mathrm{g}(\mathrm{X})=\varphi_{1}(\mathrm{X}) \varphi_{3}(\mathrm{X}) \varphi_{5}(\mathrm{X}) \\
=\left(1+\mathrm{X}+\mathrm{X}^{4}\right)\left(1+\mathrm{X}+\mathrm{X}^{2}+\mathrm{X}^{3}+\mathrm{X}^{4}\right)\left(1+\mathrm{X}+\mathrm{X}^{2}\right) \\
=1+\mathrm{X}+\mathrm{X}^{2}+\mathrm{X}^{4}+\mathrm{X}^{5}+\mathrm{X}^{8}+\mathrm{X}^{10}
\end{gathered}
$$

Therefore, the code polynomial of any $\mathrm{BCH}(\mathrm{n}, \mathrm{k})$ can be written as $c(X)=c_{0}+c_{1} X+\ldots+c_{n-1} X^{n-1}$, it has the root as following [22]:

$$
\mathrm{c}(\alpha \mathrm{i})=\mathrm{c}_{0}+\mathrm{c}_{1} \alpha^{\mathrm{i}}+\cdots+\mathrm{c}_{\mathrm{n}-1} \alpha^{\mathrm{i}(\mathrm{n}-1)}=0
$$

where

$$
\begin{gathered}
\left(c_{0}, c_{1}, \ldots, c_{n-1}\right) \circ\left[\begin{array}{c}
1 \\
\alpha^{i} \\
\alpha^{2 i} \\
\vdots \\
\alpha^{(n-1) i}
\end{array}\right]=\mathbf{0} \\
\boldsymbol{H}=\left[\begin{array}{cccccc}
1 & \alpha & \alpha^{2} & \alpha^{3} & \cdots & \alpha^{n-1} \\
1 & \alpha^{2} & \left(\alpha^{2}\right)^{2} & \left(\alpha^{2}\right)^{3} & \cdots & \left(\alpha^{2}\right)^{n-1} \\
1 & \alpha^{3} & \left(\alpha^{3}\right)^{2} & \left(\alpha^{3}\right)^{3} & \cdots & \left(\alpha^{3}\right)^{n-1} \\
\vdots & \vdots & \vdots & \vdots & & \vdots \\
1 & \alpha^{2 t} & \left(\alpha^{2 t}\right)^{2} & \left(\alpha^{2 t}\right)^{3} & \cdots & \left(\alpha^{2 t}\right)^{n-1}
\end{array}\right] \\
\\
\boldsymbol{c} \circ H^{T}=\mathbf{0}
\end{gathered}
$$

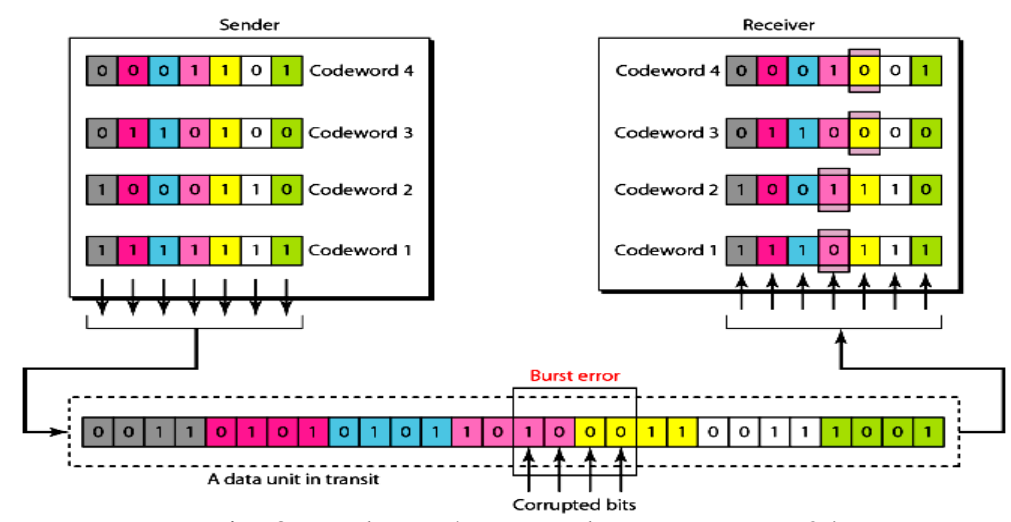

Fig. 3 Interleaver/ De-Interleaver Process [24].

$$
\boldsymbol{H}=\left[\begin{array}{cccccc}
1 & \alpha & \alpha^{2} & \alpha^{3} & \cdots & \alpha^{n-1} \\
1 & \alpha^{3} & \left(\alpha^{3}\right)^{2} & \left(\alpha^{3}\right)^{3} & \cdots & \left(\alpha^{3}\right)^{n-1} \\
1 & \alpha^{5} & \left(\alpha^{5}\right)^{2} & \left(\alpha^{5}\right)^{3} & \cdots & \left(\alpha^{5}\right)^{n-1} \\
\vdots & \vdots & \vdots & \vdots & & \vdots \\
1 & \alpha^{2 t-1} & \left(\alpha^{2 t-1}\right)^{2} & \left(\alpha^{2 t-1}\right)^{3} & \cdots & \left(\alpha^{2 t-1}\right)^{n-1}
\end{array}\right]
$$

In decoding, the received polynomial is [22]:

$$
\mathrm{r}(\mathrm{X})=\mathrm{c}(\mathrm{X})+\mathrm{e}(\mathrm{X})
$$

where, $c(X)$ and $e(X)$ are the code and error polynomials respectively, the syndrome vector is

$$
\begin{gathered}
\mathrm{S}=\left(\mathrm{s}_{0}, \mathrm{~s}_{1}, \ldots, \mathrm{s}_{2 \mathrm{t}}\right)=\mathrm{r} \circ \mathrm{HT} \\
\operatorname{si}=\mathrm{r}\left(\alpha^{\mathrm{i}}\right)=\mathrm{e}\left(\alpha^{\mathrm{i}}\right) \\
=\mathrm{r}_{0}+\mathrm{r}_{1}\left(\alpha^{\mathrm{i}}\right)+\cdots+\mathrm{r}_{\mathrm{n}-1}\left(\alpha_{\mathrm{i}}\right)^{\mathrm{n}-1}, 1 \leq \mathrm{i} \leq 2 \mathrm{t} .
\end{gathered}
$$

By using these equations and replacing the variable of $X$ by $\alpha \mathrm{i}$ root in the polynomials of received $r(X)$ to compute the $\mathrm{i}^{\text {th }}$ component of syndrome vector [22].

\subsection{Interleaver/De-Interleaver}

interleaver/De-interleaver have been applied in the suggested system to re-request input bit series to a non- adjacent technique. This alleviates the surge of burst errors in the transmission channel [23].

\subsection{Modulation Schemes}

The two lower modulations BPSK and QPSK have been selected in this study. This is due to benefits they offer with regard to increased enhancement of the LTE system performance in terms of BER and SNR compared to higher order modulation (including 16-QAM and 64QAM) that increases BER for fixed value of SNR [25].

\subsection{LTE-MIMO Channel}

The multipath LTE-MIMO channel has been applied in this study to assess the performance of the system in two scenarios of multipath fading channels, such as $(2 \times 2)$ and (4X4) transmitter and receiver antennas of MIMO channel.

The rest of parts in the proposed system such as (IFFT/FFT and Cyclic Prefix/Cyclic Prefix Removal) represent the fixed parts of OFDM system and doesn't change it in own work.

\section{Results and Discussion}

The data were analysed using MATLAB software. The curves illustrated in each figure indicates the behaviour of system performance that is relied on various circumstances. The two sides of the system performance curves are BER against signal to noise ratio (SNR). Fig. 4 
illustrates the comparison between downlink LTE system performance over LTE-MIMO channel when using BCH codes versus uncoded and other suggested coding techniques (including convolutional and turbo codes) for BPSK.

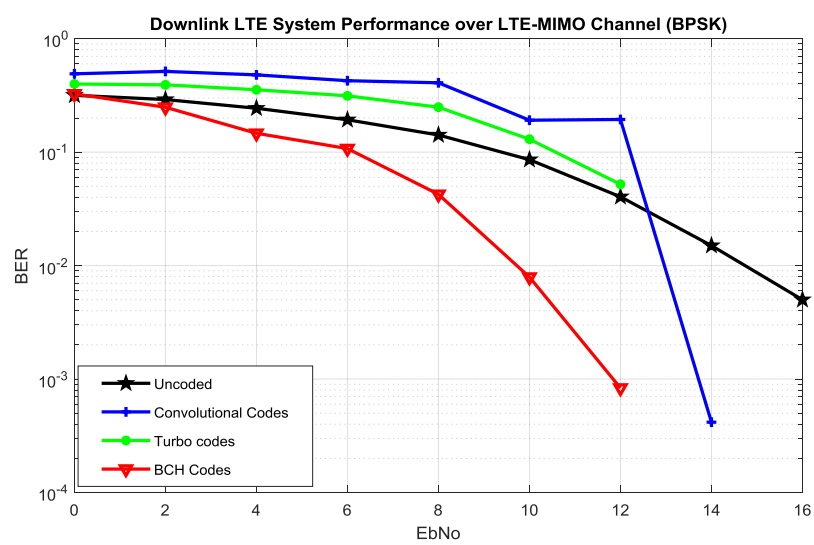

Fig. 4 The downlink LTE system performance over LTEMIMO channel (BPSK)

The findings demonstrate that; the performance of downlink LTE system when using $\mathrm{BCH}$ codes was superior compared to other suggested coding techniques. The BCH-downlink LTE system had coding gain of approximately $4 \mathrm{~dB}$ and $6 \mathrm{~dB}$ at $1 \times 10^{-1}$ versus turbo and convolutional codes, respectively. Contrarily, un-coded system had the poorest performance among the other systems. Figure 5 illustrates the comparison between downlink LTE system performance over LTE-MIMO channel when using $\mathrm{BCH}$ codes versus uncoded and other suggested coding techniques (including convolutional and turbo codes) for QPSK.

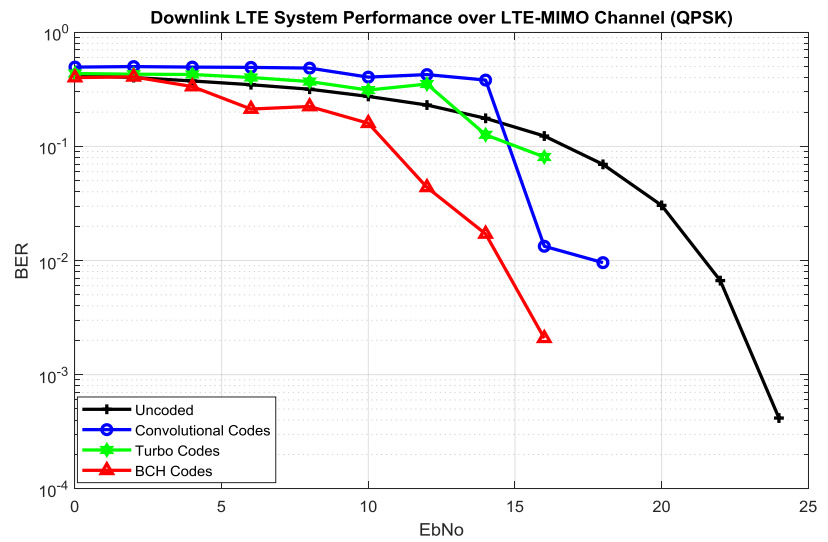

Fig. 5 The downlink LTE system performance over LTEMIMO channel (QPSK).

The findings indicated that the performance of downlink LTE system when using $\mathrm{BCH}$ codes was also superior compared to the other suggested coding techniques. The BCH-downlink LTE system had the coding gain approximately $4 \mathrm{~dB}$ at $1 \times 10^{-1}$ versus the both of convolutional and codes turbo code. In contrast, the un-coded system had also the poorest performance among the other systems. Figure 6 demonstrates the effect of utilizing BPSK versus QPSK modulation schemes on the downlink LTE system performance.

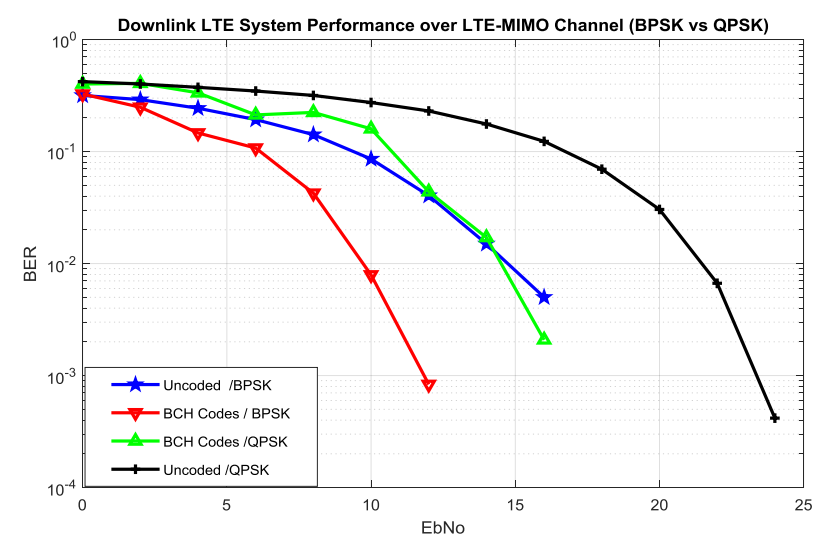

Fig. 6 The downlink LTE system performance over LTEMIMO channel (BPSK vs QPSK)

The findings revealed that; the system performance by utilizing BPSK was superior compared to QPSK modulation schemes. The BPSK had approximately $8 \mathrm{~dB}$ and $5 \mathrm{~dB}$ coding gain at $10^{-1}$ for un-coded system and $\mathrm{BCH}$ codes, respectively.

Comparison was made between (2X2) and (4X4) transmitter and receiver antennas of MIMO channel in the suggested system as illustrated the in Fig. 7.

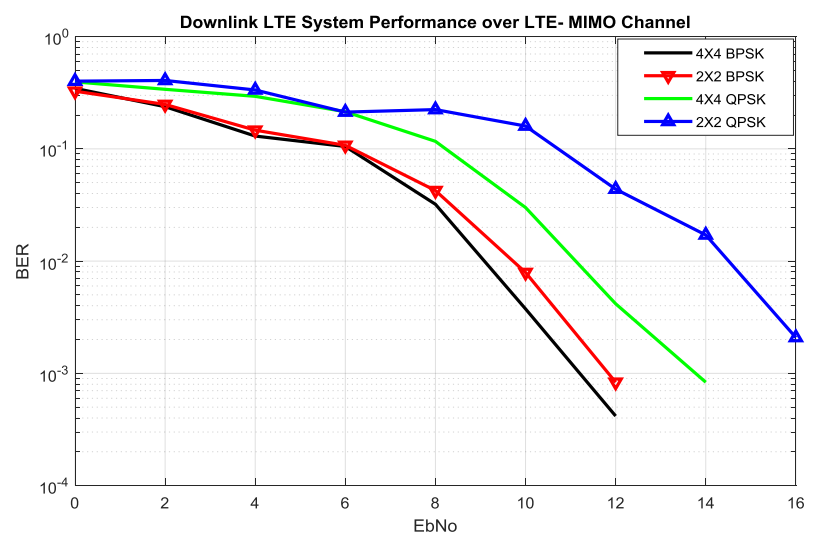

Fig. 7 The downlink LTE system performance over LTE-MIMO channel

The comparison indicates further enhancement with respect to the performance of the suggested system with the increase in the number of antennas in MIMO channel for both of BPSK and QPSK. Moreover, the BER of the received data was diminished through increasing in the number of antennas in the MIMO channel from (2X2) to (4X4) as illustrated in Fig. 7. In order to enhance the system performance, there will be a trade-off between the number of antennas in the MIMO channel and the total deployment cost. Therefore, it is important to achieve a balance between the optimal number of antennas and reasonable deployment cost. 


\section{Conclusion}

The current study evaluated the downlink LTE system performance in multipath fading channel. The findings revealed that the system performance is enhanced with the use of $\mathrm{BCH}$ codes over LTE-MIMO channel. The merging of $\mathrm{BCH}$ codes and MIMO channel within LTE system has shown to enhance the system performance and capacity. By using BCH codes with LTE system versus both convolutional and turbo codes, the system performance was improved through reduction of BER meant for both modulation schemes namely BPSK and QPSK. Moreover, increase in the number of antennas in MIMO-channel to (4X4) caused reduction in the BER of received data. Therefore, the proposed method contributed in the improvements of the Downlink LTE system performance in presence of multipath fading environments, when the BER for both of BPSK and QPSK are reduced by using $\mathrm{BCH}$ code that have lower decoding complexity than both of convolutional and turbo codes. Essentially, the future studies should investigate the trade-off between decoding complexity and enhancement of systems with respect to their application in LTE system for attaining error control system. As error correction methods are advantageous, other types of error correction methods should also be explored for broader applications.

\section{Acknowledgments}

This research was funded by the Ministry of Higher Education Malaysia under Fundamental Research Grant Scheme Vot No. 1627 and partially sponsored by Universiti Tun Hussein Onn Malaysia.

\section{References}

[1] Mousavi, H., Amiri, I. S., Mostafavi, M. A., Choon, C. Y. LTE physical layer: Performance analysis and evaluation. Science Direct, Applied Computing and Informatics, (2017).

[2] Tsolkas, D., Liotou, E., Passas, N., Merakos, L. Ltea access, core, and protocol architecture for $\mathrm{d} 2 \mathrm{~d}$ communication, in: Smart Device to Smart Device Communication, Springer International Publishing Switzerland, (2014), pp. 23-40.

[3] Sesia, S., Toufik, I. and Baker, M. LTE-The UMTS Long Term Evolution: From Theory to Practice. United Kingdom: John Wiley \& Sons Ltd, (2011). ISBN 978-0-470-66025-6

[4] Nguyen, V. G., Kim, Y. Proposal and evaluation of SDN-based mobile packet core networks. EURASIP Journal on Wireless Communications and Networking, a Springer Open Journal, (2015), pp. $1-18$.

[5] Dixit, S., Katiyar, H. Performance of OFDM in time selective Multipath fading channel in $4 \mathrm{G}$ Systems. Communication Systems and Network Technologies (CSNT), 2015 Fifth International Conference, (2015), pp. 421-424.

[6] Thakur, R. S., Jain, S. Advanced Techniques in MIMO-OFDM Systems for LTE Environment. International Journal on Emerging Technologies, Volume 6, (2015), pp. 15-19.

[7] Ajey, S., Srivalli, B., Rangaraj, G. V. On Performance of MIMO-OFDM Based LTE Systems. IEEE, International Conference on Wireless Communication and Sensor Computing, ICWCSC. Chennai, India, (2010).

[8] Zarei, S. LTE: Der Mobilfunk der Zukunft, Channel Coding and Link Adaptation. Seminar:Ausgewählte Kapitel der Nachrichtentechnik, 16, (2009).

[9] Elwazan, A. A. E., Zekry, A. A. A., Zayed, H. L. A. Matlab Code for LTE Convolutional Code and Viterbi Decoder. International Journal of Engineering Research \& Technology (IJERT), Volume 6, (2017), pp. 578-581.

[10] Singh, S., Rathore, S. S. Performance Comparision of Coded and Un-Coded OFDM for Different Fic Code. Journal of Engineering Research and Applications, Volume 4, (2014), pp.

[11] Anghel, C., Paleologu, C., Stanciu, C. Performances Evaluation of CTC Turbo Decoder for LTE Systems. 57th International Symposium ELMAR-2015, Zadar, Croatia, (2015).

[12] Li, A., Xiang, L., Chen, T., Maunder, R., AlHashimi, B. L. HANZO. VLSI Implementation of Fully Parallel LTE Turbo Decoders. IEEE Journals \& Magazines, Volume 4, (2016), pp. 323 - 346.

[13] Bajoga, B. G., Walbesser, W. J. Decoder complexity for $\mathrm{BCH}$ codes. IEEE, Proceedings of the Institution of Electrical Engineers, Volume 120, (1973), pp. 429-431.

[14] Alwan, M. H.,Singh, M., Mahdi, H. F. Performance comparison of turbo codes with LDPC codes and with $\mathrm{BCH}$ codes for forward error correcting codes. IEEE Student Conference on Research and Development (SCOReD), Kuala Lumpur, Malaysia, (2015), pp. 556-560.

[15] 3GPP TS 36.101 version 10.3.0 Release 10. LTE; Evolved Universal Terrestrial Radio Access (EUTRA); User Equipment (UE) radio transmission and reception. France. European Telecommunications Standards Institute. 2011.

[16] Bose, R. C., Ray-Chaudhuri, D. K. On a class of error correcting binary group codes. Elsevier, Science Direct, Information and Control, Volume 3, (1960), pp. 68-79.

[17] Bose, R. C., Ray-Chaudhuri, D. K. Further results on error correcting binary group codes. Elsevier, 
Science Direct, Information and Control, Volume 3, (1960), pp. 279-290.

[18] Hocquenghem, A. Codes correcteurs d'erreurs, Chiffres, (Paris) 2, (1959), pp.147-156.

[19] Skupien, Z. BCH codes and distance multi- or fractional colorings in hypercubes asymptotically. Elsevier, Science Direct, (2007), pp. 990 - 1000.

[20] Skupien, Z. Some maximum multigraphs and edge/vertex distance colourings. Discuss. Math. Graph Theory 15,(1995), pp. 89-106.

[21] Zolghadrasli, A., Ghamat, M. H. A New Blind PAPR Reduction and Error Correction Method in OFDM System Using $\mathrm{BCH}$ Codes. IEEE International Conference on Signal Processing and Communications (ICSPC 2007), Dubai, United Arab Emirates, (2007).

[22] Moreira, J. F., Farrell, P. G. ESSENTIALS OF ERROR-CONTROL CODING. Chichester: John
Wiley \& Sons Ltd. (2006).

[23] Han, Y., Harliman, P., Kim, S. W., Kim, J., Kim, C. A Novel Architecture for Block Interleaving Algorithm in MB-OFDM Using Mixed Radix System. IEEE Transactions on Very Large Scale Integration (VLSI) Systems, Volume 18, (2010), pp. 1020 - 1024.

[24] Behrouz, A. F. Data Communication and Networking. New York: McGraw-Hill. (2007). ISBN. 978-0-07-296775-3.

[25] Sukar, M. A. N., Pal, M. SC-FDMA \& OFDMA in LTE physical layer. International Journal of Engineering Trends and Technology (IJETT), Volume 12, (2014), pp. 74-85. 